\title{
Submucosal territory of the direct lymphatic drainage system to the thoracic duct in the human esophagus
}

\author{
Kenshi Kuge, $M D^{a}$ \\ Gen Murakami, MD \\ Shunji Mizobuchi, MD \\ Yoichi Hata, $\mathrm{MD}^{\mathrm{c}}$ \\ Takashi Aikou, MD ${ }^{\mathrm{c}}$ \\ Shiro Sasaguri, MD
}

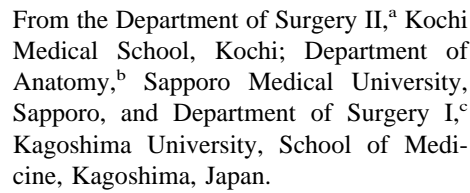

This work is partly supported by a Grantin-Aid for Scientific Research (No. 12670024) from the Ministry of Education, Science and Culture of Japan.

Received for publication Feb 11, 2002; revisions requested April 30, 2002; revisions accepted Aug 18, 2002; accepted for publication Sept 11, 2002.

Address for reprints: Gen Murakami, MD, $\mathrm{PhD}$, Department of Anatomy, Sapporo Medical University School of Medicine, South 1, West 17, Sapporo, 060-8556 Japan (E-mail: chisa@sapmed.ac.jp).

J Thorac Cardiovasc Surg 2003;125:1343-9

Copyright (C) 2003 by The American Association for Thoracic Surgery

0022-5223/2003\$30.00+0

doi:10.1016/S0022-5223(03)00036-9
Objective: To investigate how large submucosal drainage territory extends in lymphatic drainage vessels of the esophagus with and without nodal delay and which morphologies are shown when passing through the muscularis propria.

Methods: Submucosal territories of the 22 highly selected direct drainage vessels of 17 esophagi were histologically examined using transverse or sagittal serial sections. Afferent vessels from the esophagus to the subcarinal (6 esophagi) and para-esophageal ( 5 esophagi) nodes were also examined to identify their courses and drainage territories.

Results: We found the direct drainage vessel from the esophagus in 17 of 75 cadavers macroscopically $(22.7 \%)$. A single submucosal drainage unit gave off 1-3 thick drainage vessels passing through a complete muscle gap of the 2 muscular layers. The unit extended longitudinally for $>40 \mathrm{~mm}$ but was restricted to the right and/or dorsal quadrants of the esophagus. In contrast, drainage routes with a nodal relay originated from the intermuscular area, except 1 case when the adjacent or concomitant esophageal artery and vein provided the complete muscle gap.

Conclusions: Due to the extended longitudinal but restricted transverse territory of the direct drainage system without a nodal relay and because of the suggested much more frequent occurrence in patients than in cadavers, when superficial carcinoma is found in the dorsal and/or right quadrants of the esophagus, we recommend detailed presurgical investigations of cervical nodes. In contrast, afferents from the esophagus to the first regional node usually seemed to be less responsible for early nodal metastasis than the direct drainage route because of their intermuscular origins.

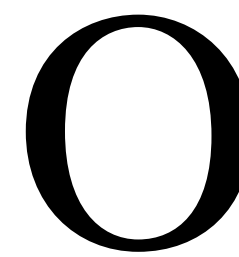

ne of the authors (G.M.) histologically described the lymphatic drainage vessel originating from the human esophagus and merging with the thoracic duct directly without a nodal relay. ${ }^{1}$ At almost the same period, Riquet and colleagues ${ }^{2}$ identified the direct drainage flow from the esophagus to the thoracic duct using an injection method. Recently, Rice and colleagues ${ }^{3}$ and Rice ${ }^{4}$ presented an excellent diagram showing esophageal lymphatic anatomy including the intra-esophageal configuration. To the best of our knowledge, however, no anatomical investigations have demonstrated how large the submucosal drainage territory extends. Treatment of superficial esophageal carcinoma is one of the current topics in esophageal surgery, ${ }^{3,5-7}$ especially, in sentinel node navigation surgery (SNNS). ${ }^{8}$ The aim of this research is to describe a unit of the submucosal territory of 1 lymphatic drainage vessel of the thoracic esophagus not only when it 
drains into the thoracic duct without a nodal relay but also when it does into the esophageal regional node.

\section{Materials and Methods}

Seventy-five postmortemly treated cadavers dissected in dissection practice in 2001 for medical or dental students at Sapporo Medical University, the Health Sciences University of Hokkaido, and Tokyo Medical and Dental University were used.

\section{Defining the Drainage Territory of 1 Lymphatic Vessel Without a Nodal Relay}

In 17 of the 75 cadavers (22.7\%), we found macroscopically 22 lymph vessels exiting from the esophagus (1 vessel in each of 13 cadavers, 2 in each of 3 cadavers, and 3 in 1 cadaver). These cadavers did not carry macroscopical tumors in the esophagus and upper abdominal viscera. The distribution of the 22 vessels as defined by the level of the vertebral body and its lower-adjacent disc was 2 vessels at T2, 2 at T3, 3 at T4, 4 at T5, 8 at T6 and, 3 at T7. The thickness of these vessels ranged from $0.5-1.5 \mathrm{~mm}$ (0.5-0.9 $\mathrm{mm}$ in 12 vessels and $1-1.5 \mathrm{~mm}$ in 10).

Those vessels were labeled by carbon particle suspension at the exit point on the esophagus. The 17 esophagi were cut and removed as long pieces (40-70 $\mathrm{mm}$ in length) to include an area $20-\mathrm{mm}$ oral and anal at minimum from the exit point into the piece. Thus, when 1 vessel was included in a specimen, the piece was 40 $\mathrm{mm}$ in length. After routine preparation of paraffin sections, the histological specimens were prepared and were stained with hematoxylin and eosin. Fourteen esophagi, including 19 vessels, were used for serial transverse sections ( $10 \mu \mathrm{m}$ in thickness), while 3 esophagi (each contained 1 vessel) were processed for serial sagittal sections.

\section{Defining the Drainage Territory of 1 Lymphatic Vessel to a Regional Node}

In the 75 cadavers, we chose 6 esophagi with a thick afferent vessel from the esophagus to the subcarinal node. The thickness of these vessels ranged from $0.5-1.5 \mathrm{~mm}$. We also chose another 5 esophagi with the thoracic para-esophageal node (a node attaching on the esophagus). Those 11 specimens were processed for histology in the same manner for the direct drainage (see above), but all 11 were used for serial transverse sections (10 $\mu \mathrm{m}$ in thickness).

We estimated reduction in scale to $75 \%$ due to fixation and dehydration in the present routine histology using paraffin sections, according to our previous scale comparisons of a same specimen of the esophagus among fresh (6 hours after death), formalin-fixed, and paraffin-embedded conditions (detailed data, not shown).

\section{Results}

\section{Direct Lymphatic Drainage Vessel From the Esophagus to the Thoracic Duct}

The direct drainage was found in $22.7 \%$ of 75 esophagi. All 22 vessels were counted. According to histology, however, 7 (5 esophagi) of the 22 vessels (17 esophagi) contained red blood cells in their lumina. Although venous reflux into the thoracic duct was likely due to high pressure at the injection of formalin solution for the postmortem treatment of cadav- ers, ${ }^{9}$ for strict identifications, these 7 vessels or 5 esophagi were excluded from the present study to identify the drainage territory. Consequently, 12 vessels (9 esophagi) were investigated using transverse sections, while 3 other vessels (3 esophagi) were used for sagittal sections. Observations of these 15 vessels are described.

At the exit point of the direct drainage vessel on the esophagus, which was labeled with a carbon particle suspension during macroscopic observations to choose histological specimens (Figure 1), we observed the muscle gap ${ }^{10}$ through which the vessel passed. The muscle gaps were divided into complete gaps (a large canal of 2 muscle layers of the muscularis propria) and incomplete gaps (a narrow slit of the outer longitudinal muscularis propria). The complete gap, a straight tube-like space piercing through the 2 muscle layers, contained the thick drainage vessel connecting the submucosal and external lymphatic vessels of the esophagus. These exit points or gaps were located at the dorsal and/or right aspect of the esophagus. Five of the 8 relatively thin $(0.5-0.9 \mathrm{~mm})$ vessels and all 7 of the thick (1-1.5 mm) vessels passed through a complete muscle gap (1 vessel for 1 gap). This gap ranged from $0.5-2.0 \mathrm{~mm}$ in the transverse axis and $0.2-0.4 \mathrm{~mm}$ in the longitudinal axis. In contrast, another 3 relatively thin vessels originated at the intermuscular layer and passed through an incomplete gap smaller (0.1-0.3 $\mathrm{mm}$ in the transverse axis and 0.1-0.2 mm in the longitudinal axis) than the complete one.

In the complete muscle gap, the lymphatic vessel almost always ran solitarily without a concomitant artery and/or vein. The lymphatic vessel had a thick wall $(30-50 \mu \mathrm{m})$ containing much smooth muscle and an expanded lumen (0.1-0.5 mm), showing a very tortuous arrangement (Figure 1). In contrast, in the incomplete gap, the wall and lumen were thin and the vessel was straight (Figure 1).

When the complete gap was present, the submucosal lymphatic plexus was developed well between the muscularis mucosae and inner circular muscularis propria. Notably, the plexus had a thick wall with much smooth muscle (20-30 $\mu \mathrm{m})$, in contrast to the walls of neighboring veins (5-10 $\mu \mathrm{m})$, the lumina of which were filled with red blood cells. The plexus extended longitudinally over $20-\mathrm{mm}$ oral and anal from the level of the complete gap, maintaining a luminal thickness of more than $50 \mu \mathrm{m}$ (Figures 2 and 3). Thus, the longitudinal length of the drainage territory was identified as at least $40 \mathrm{~mm}$ according to the criterion of the 50- $\mu \mathrm{m}$ minimum luminal thickness.

In the transverse axis, the plexus was identified in restricted, dorsal, and/or right quadrants of the esophagus, ranging from 5 to 11 o'clock points (Figures 1 and 3), corresponding to 1 or 2 mucosal folds, although the position varied in the long longitudinal configuration of the plexus. The transverse width of the territory ranged from $4-10 \mathrm{~mm}$. Consequently, the submucosal lymphatic territory corre- 


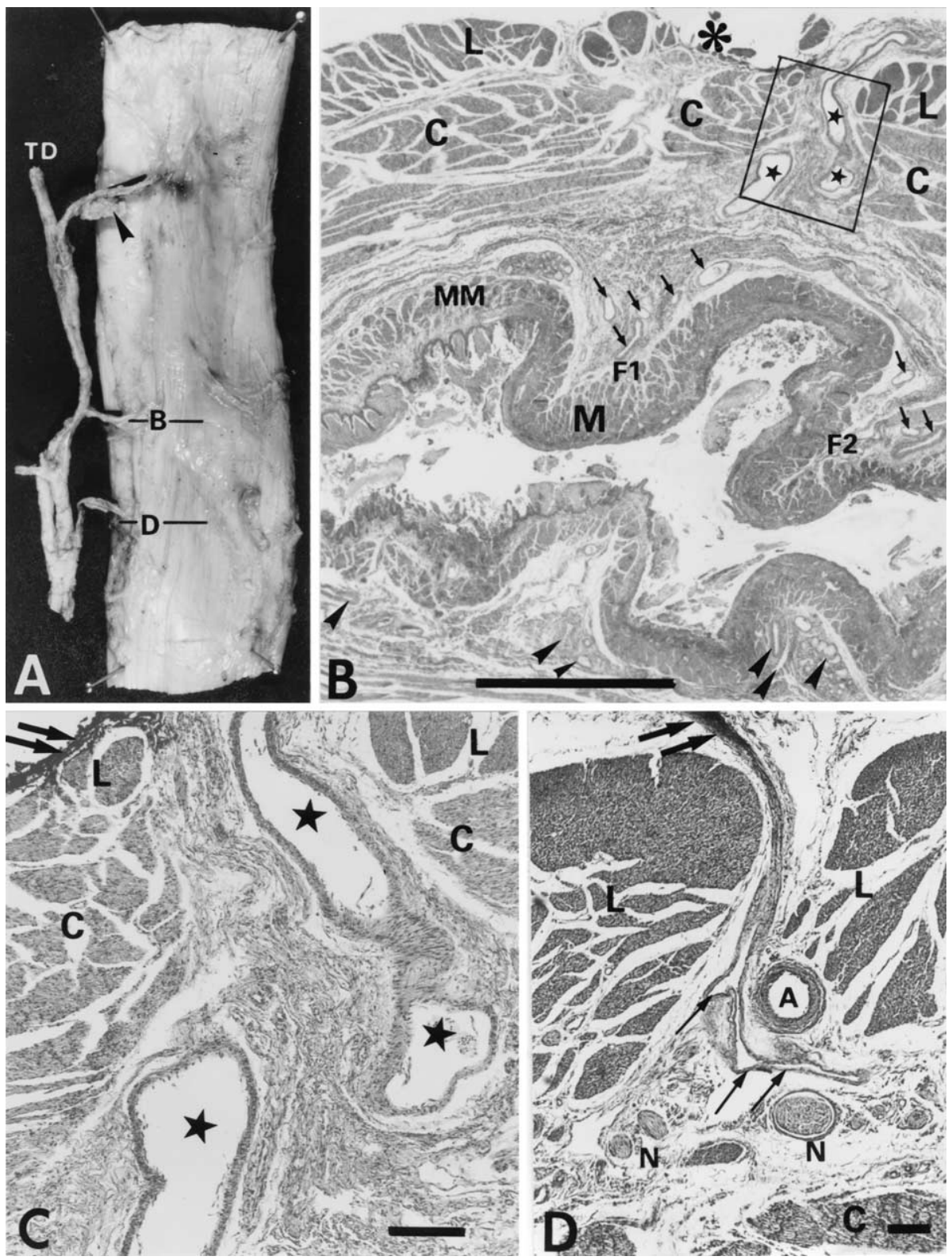

Figure 1. Direct lymphatic drainage from the esophagus to the thoracic duct: anatomical and cross-sectional representations. A, B, C, and D display the same specimen. A, Dorsal aspect of the thoracic esophagus. The thoracic duct (TD) receives 3 macroscopically identified drainage vessels from the esophagus. The most cranial vessel (T4 level) is associated with a small lymph node (arrowhead). B, Cross section along the plane indicated by -B- in (A) (T5 level). The outer longitudinal $(L)$ and inner circular $(C)$ muscle layers are interrupted by a complete muscle gap through which a thick tortuous vessel (stars) passes. An asterisk indicates a damaged area during the procedure. Submucosal lymph vessels (small arrows) are observed at the base of the mucosal folds (F1, F2). According to tracking of transversely directional continuations using the serial sections, dorsal vessels (small arrows) communicate with the tortuous drainage vessel, but other ventral vessels (arrowheads) do not. The mucosal layer $(M)$ includes thick muscularis mucosa (MM). Bar: $10 \mathrm{~mm}$. C, Higher magnification view of the square in (B). The drainage vessel was labeled by carbon particles (large arrows) during macroscopic observations. Note the thick wall $(20-50 \mu \mathrm{m})$ comprised of the smooth muscle layer. The intima of the vessel is not preserved in this case. Bar: $100 \mu \mathrm{m}$. D, Incomplete muscle gap. Cross section along the plane indicated by $-\mathrm{D}$ - in (A). A straight drainage vessel originates from the intermuscular space. Its transverse continuation ( 3 thin arrows) was limited. Two thick arrows indicate a carbon-particle label during macroscopical identification. $A$, artery; $\boldsymbol{N}$, myenteric nerve plexus of Auerbach. Bar: $100 \mu \mathrm{m}$. 


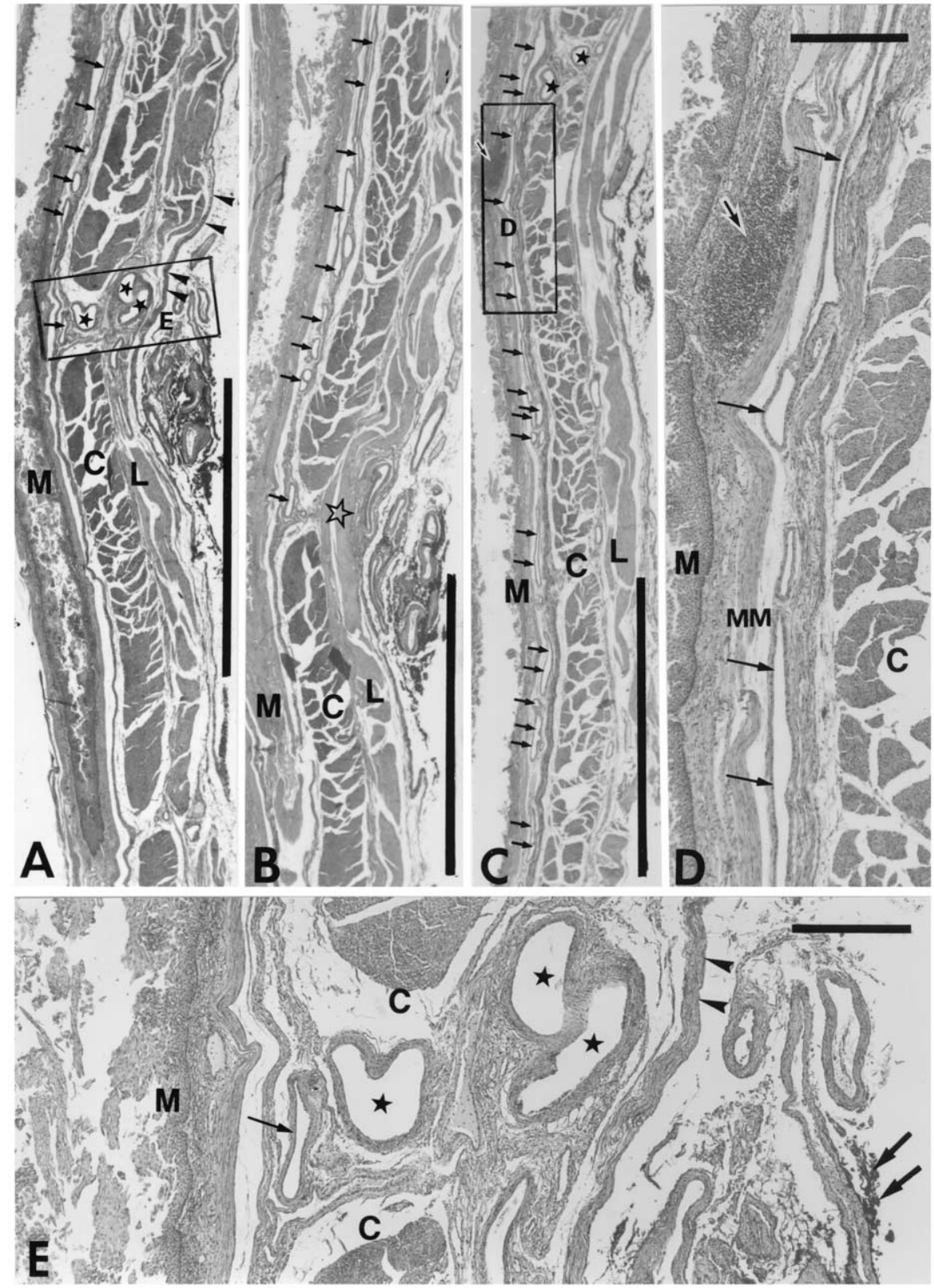

Figure 2. Longitudinal territory of 1 drainage vessel running through the complete muscle gap. A, B, and C correspond to 3 of the serial sagittal sections of the same specimen, while their higher magnification views are shown in (D) and (E). A, Level of the complete muscle gap through which a tortuous drainage vessel (stars) runs. In this section, the gap is covered by a definite layer of connective tissue such as the adventitia (arrowheads). Note cranially extended submucosal vessels (small arrows). Those vessels communicate with each other and with the drainage vessel through the gap in another section. $L$ and $C$, outer longitudinal and inner circular muscle layers. $M$, mucosal layer. Bar: $10 \mathrm{~mm}$. B, Sagittal level $2 \mathrm{~mm}$ right side of (A). The longitudinal muscle layer $(L)$ is continuous, but the circular layer still forms a small gap (open star). The submucosal vessels (small arrows) extend cranially more than $20 \mathrm{~mm}$ from the gap. Bar: $10 \mathrm{~mm}$. C, Sagittal level $4 \mathrm{~mm}$ left of (A). The muscle gap can still be recognized as a gap of the circular layer and thick, tortuous vessel (stars). Note caudally extended vessels (small stars) in the submucosal layer. D, a higher magnification view of the square with $D$ in (C). Note a lymph follicle-like structure (black and white arrow) that is separated by the muscularis mucosae (MM) from the submucosal lymphatic vessels. Four small arrows correspond to those in (C). Bar: $1 \mathrm{~mm}$. E, a higher magnification view of the complete gap, including the tortuous drainage vessel [a square with $E$ in $(A)$ ]. Note the thick, smooth muscle wall $(50-100 \mu \mathrm{m})$ of the vessel. Thick arrows indicate carbon particles labeled during macroscopic observations. The muscle gap is covered by a connective tissue layer (arrowheads). Bar: 1 mm. 
sponding to 1 complete gap or 1 direct drainage vessel was determined to have a size of $4-10 \mathrm{~mm}$ transversely and $>40$ mm craniocaudally.

The intramuscular drainage territory was difficult to identify when the vessel was thin and passing through the incomplete gap because longitudinal and transverse continuation of the intramuscular lymphatics could not be recognized even in serial sections. In spite of the insignificant morphology, however, we did not ignore the incomplete muscle gap because "usual" drainage with a nodal relay seemed to usually accompany the incomplete gap (see below). No definite valves were found in the submucosal plexus with its efferent passing through the complete muscle gap.

\section{Lymphatic Drainage From the Esophagus With a Nodal Relay}

To determine the drainage territory of the subcarinal node and para-esophageal node, 6 candidates for afferent vessels from the esophagus to the subcarinal node (6 esophagi) and other 5 para-esophageal nodes (another 5 esophagi) were examined histologically (photographs not shown). The candidate vessels to the subcarinal node usually ( 5 of 6 ) originated at the intermuscular layer and passed through the incomplete gap, but rarely (1 of 6), passed through the complete gap with its concomitant artery and vein to communicate with the submucosa. In 1 rare case, the submucosal histology was similar to that in the direct drainage cases. Although using serial sections, because of the very tortuous course and nonextended lumen, we found no definite afferent vessel terminating the marginal sinus of the para-esophageal node. Consequently, except for the esophagus in which the feeding artery and its concomitant vein provided the complete muscle gap, a route with a nodal relay was likely to originate at the intermuscular area.

\section{Discussion}

Identification of peripheral lymphatic vessels is usually thought to be possible by specific histochemical methods. ${ }^{11-13}$ However, we believe that its direct connection to the thoracic duct is, if observed even on hematoxylin and eosin staining, a simple but primary criterion for the identification. Although it was limited to a specific lymphatic drainage pattern (ie, the direct drainage without a nodal relay) and although surgeons might characterize the intraesophageal lymphatics by the occurrence of mucosal lymph capillaries, ${ }^{3,4}$ the present research first photographically demonstrated an almost complete set of the esophageal lymphatic routes from the submucosal origin to its terminal at the thoracic duct. Notably, in contrast to the usual lymphatic plexi (ie, capillary meshwork) reported in the diaphragm, ${ }^{14}$ omentum, ${ }^{15}$ subpleural tissue,${ }^{16}$ and subcutaneous tissue, ${ }^{17}$ the submucosal lymphatic origin in the thoracic

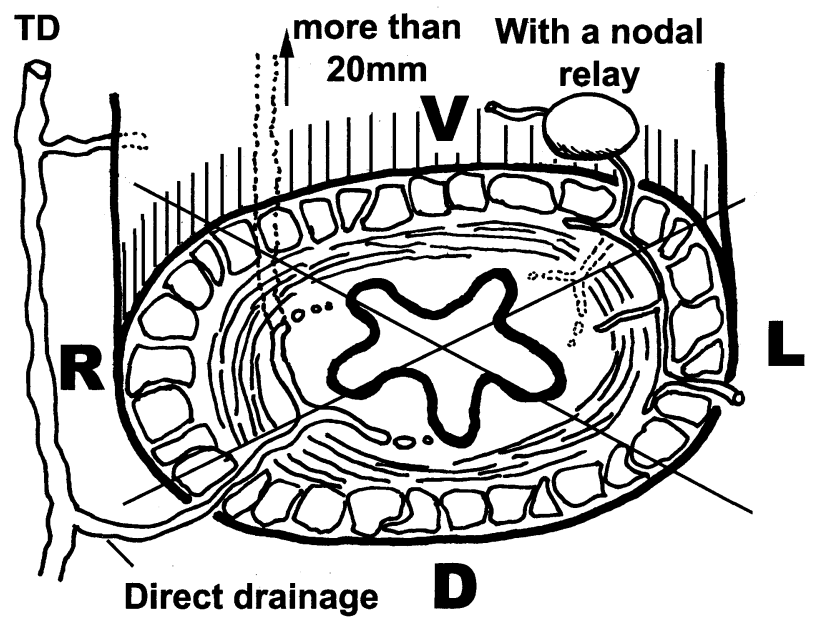

Figure 3. Schematic representation showing configurations of the lymphatic drainage from the esophagus. A thick, direct drainage vessel originates from the submucosal lymphatic plexus and passes through the complete muscle gap in the dorsal and/or right quadrants $(D$ and/or $R$ ) of the esophagus. For another thin vessel draining toward the regional node, an incomplete gap is formed at the longitudinal layer of the muscularis propria. $V$ and $L$ indicate the ventral and left quadrants, respectively. TD, Thoracic duct.

esophagus was composed of thick vessels containing a well-defined smooth muscle layer (ie, not capillaries). Moreover, the submucosal lymphatic vessels were much more numerous and thicker in the thoracic esophagus than in the cervical esophagus. ${ }^{18}$ The long longitudinal arrangement of the thick submucosal vessels (even $>50 \mu \mathrm{m}$ in diameter) was absolutely consistent with the classical physiological hypothesis that the longitudinal lymph flow is 6 times as great as the transverse flow in the esophagus. ${ }^{19}$

The complete muscle gap was an evident morphology. The gap was much larger than the draining vessel passing through it and provided enough space for the vessel to run tortuously. In contrast to the direct drainage route, esophageal lymphatic routes with a nodal relay usually originate from the intermuscular area of the muscularis propria and pass through the incomplete muscle gap. Lymphatic communication between the submucosal and intermuscular areas was not identified histologically. When the complete gap is present adjacent to a node for the artery and vein, the node would be easily identified as a sentinel node by local injection and, moreover, as a positive node in the early stage. Nevertheless, we should remember that the direct drainage route and its long extended territory are the critical limiting factor for SNNS. This route may not involve any nodes. However, identification of the large muscle gap seems to be a challenging work with a specific technology, 
such as an endothoracic scope using an ultrared lamp in combination with injection of indocyanine green.

Kato and colleagues ${ }^{5}$ reported that in $57.6 \%$ of 43 patients with superficial esophageal squamous carcinoma, lymph node metastasis was evident and that $15.2 \%$ of the submucosal cases had positive nodes in the neck. The likely distant transportation of cancer cells by the direct lymphatic drainage route from the submucosal plexus seems to be responsible for those cervical metastases. The direct lymphatic drainage to the thoracic duct has been identified macroscopically in cadavers with limited incidences (19.0\% by Murakami and colleagues ${ }^{1} ; 22.7 \%$ in the present research). However, the direct drainage vessel is likely to be found much more frequently in the living state than in cadaveric specimens in which postmortem necrotic changes occur and progress. Therefore, recent advancements in micrometastasis research using cytokeratin immunohistochemistry in association with systematic lymph node dissection would provide incidences of early neck metastasis coinciding with the anatomical evidence. ${ }^{20}$ Kato and colleagues ${ }^{5}$ also reported that superficial esophageal squamous carcinoma brought no metastasis to the subcarinal node. Therefore, the single specimen found in the present work with the afferent from the esophageal submucosa to the node seemed to be a rare exception with direct drainage. Because the afferent usually originated at the intermuscular layer of the muscularis propria, even submucosally invasive squamous carcinoma may provide no early metastasis through the afferent (see also above discussion about SNNS).

Notably, the direct drainage territory appeared to be restricted to the right and/or dorsal quadrants of the thoracic esophagus (Figure 3), although the "end" of the submucosal plexus was defined by its diameter $<50 \mu \mathrm{m}$ in the present research. Thus, the topographical relation between the thoracic duct and esophagus seemed to be an essential factor in determining the position of the direct drainage territory. Therefore, when superficial carcinoma is found in the dorsal and/or right quadrants, the limited incidence of direct drainage, ie, about $20 \%$ or "much more" (see above), seems to correspond to the actual value rather than an overevaluation. When superficial carcinoma is found in the dorsal and/or right quadrants of the esophagus, even without submucosal invasion, we recommend detailed presurgical investigations of cervical lymph nodes, which are likely to receive the early cancer metastasis from the anatomically distant thoracic esophagus through the thoracic duct. Moreover, the long longitudinal extension of the drainage territory was very evident in the esophageal submucosa. This also seemed to be responsible for unexpected lymph node recurrence after endoscopic mucosal resection of superficial esophageal carcinoma, although indications for the endoscopic technique might extend to even submucosal carcinoma.

\section{Study Limitation}

First, a specific morphology (ie, direct drainage) might not be combined with the "usual" configuration of the submucosal lymph plexus, such as the usual size of the drainage territory. When considering finding the direct drainage in 12 of 75 esophagi, possible selection bias should not be ignored. Second, all histological specimens were too small in length to identify the real morphology. We underestimated the drainage territory (or an area in which dilated lumen was observed) as restricted to an about 20 -mm range from the muscle gap. However, it extended much further than expected. Third, we conventionally defined the end of the plexus as the point at which the luminal thickness became $<50 \mu \mathrm{m}$. However, its dilation or shrinkage seemed to depend on physiology and even on conditions at and after death. Fourth, in spite of the meticulous work to prepare serial sections, we could obtain only a few photographs demonstrating a connection between the para-esophageal node and intra-esophageal lymphatics. Moreover, identification of these afferent lymph vessels, including those of the subcarinal node, was only based on the fact that they connected to the marginal sinus of the node. However, the marginal sinus was often closed or not clearly identified due to severe anthracosis. Finally, the present results seemed to be insufficient to reveal the mechanism of frequent metastasis of superficial esophageal carcinoma to the perigastric node,${ }^{5,6}$ because our materials did not include the lowermost thoracic esophagus. Moreover, the well-known, high incidence of early metastasis to the recurrent nerve node ${ }^{5,6}$ might not occur via direct drainage even considering lymph reflux. Japanese surgeons are now discussing the reliability of the recurrent nerve node as a critical sentinel node to determine whether the patient needs cervical lymphadenectomy in superficial esophageal squamous carcinoma, ${ }^{20}$ although the direct drainage seems to be contrary to the sentinel node concept because there is no nodal relay. Nevertheless, we believe that the present study successfully describes a critical aspect of the esophageal lymphatic drainage.

We are grateful to Professor Toshihiko Yajima, Department of Anatomy, Health Sciences University of Hokkaido, and Professor Tatsuo Sato, Department of Functional Morphology, Graduate School of Tokyo Medical and Dental University, for their permission to use their materials. We also thank the teaching and technical staffs in those universities for their kind assistance.

\section{References}

1. Murakami G, Sato I, Shimada K, Dong C, Kato Y, Imazeki T. Direct lymphatic drainage of the esophagus to the thoracic duct. Surg Radiol Anat. 1994;16:399-407.

2. Riquet M, Saab M, LePimpec-Barthes F, Hidden G. Lymphatic drainage of the esophagus in the adult. Surg Radiol Anat. 1993;15:209-11.

3. Rice TW, Zuccaro G Jr, Adelstein DJ, Rybicki LA, Blackstone EH, 
Goldblum JR. Esophageal carcinoma: depth of tumor invasion is predictive of regional lymph node status. Ann Thorac Surg. 1998;65: 787-92.

4. Rice TW. Superficial oesophageal carcinoma: is there a need for three-field lymphadenectomy? Lancet. 1999;354:792-4.

5. Kato H, Tachimori Y, Mizobuchi S, Igaki H, Ochiai A. Cervical, mediastinal, and abdominal lymph node dissection (three-field dissection) for superficial carcinoma of the thoracic esophagus. Cancer. $1993 ; 72: 2879-82$.

6. Matsubara T, Ueda M, Abe T, Akimori T, Kokudo N, Takahashi T. Unique distribution patterns of metastatic lymph nodes in patients with superficial carcinoma of the thoracic esophagus. Br J Surg. 1999;86: 669-73.

7. Nishimaki T, Suzuki T, Kanda T, Obinata I, Komukai S, Hatakeyama K. Extended radical esophagectomy for superficially invasive carcinoma of the esophagus. Surgery. 1999;125:142-7.

8. Kitagawa Y, Fujii H, Mukai M, Kubota T, Ando N, Watanabe M, et al. The role of the sentinel lymph node in gastrointestinal cancer. Surg Clin North Am. 2000;80:1799-809.

9. Murakami G, Sato T, Takiguchi T. Topographical anatomy of the bronchomediastinal lymph vessels: their relationships and the formation of the collecting trunks. Arch Histol Cytol.. 1990;53(Suppl):21935 .

10. Murakami G, Abe M, Abe T. Last-intercalated node and direct lymphatic drainage into the thoracic duct from the thoracoabdominal viscera: a review. Jpn J Thorac Cardiovasc Surg. 2002;50:93-103.

11. Kato S. Enzyme-histochemical identification of lymphatic vessels by light and backscattered image scanning electron microscopy. Stain Technol. 1990;65:131-7.

12. Schmelz M, Franke WW. Complexus adhaerentes, a new group of des1moplakin-containing junctions in endothelial cells: the syndesmos connecting endothelial cells of lymph nodes. Eur J Cell Biol. 1993; 61:274-89.

13. Schmelz M, Moll R, Kuhn C, Franke WW. Complexus adhaerentes, a new group of desmoplakin-containing junctions in endothelial cells: II. Different types of lymphatic vessels. Differentiation. 1994;57:97-117.

14. Ohtani Y, Ohtani O, Nakatani T. Microanatomy of the rat diaphragm: a scanning and confocal laser scanning microscopic study. Arch Histol Cytol. 1993;56:317-28.

15. Shimotsuma M, Shields JW, Simpson-Morgan MW, Sakuyama A, Shirasu M, Hagiwara A, Takahashi T. Morpho-physiological function and role of omental milky spots as omentum-associated lymphoid tissue (OALT) in the peritoneal cavity. Lymphology. 1993;26:90-101.

16. Masada S, Ichikawa S, Nakamura Y, Uchino S, Kato H. Structure and distribution of the lymphatic vessels in the parietal pleura of the monkey as studied by enzyme-histochemistry and by light and electron microscopy. Arch Histol Cytol. 1992;55:525-38.

17. Noshida S, Ohkuma M. Enzyme-histochemical identification of the human cutaneous lymphatics by adenylate cyclase. Lymphology. 1992; 25:184-90.

18. Nakazawa T, Nishikawa M, Aikawa E, Kasajima T. Lymph flow in the wall of the human cervical esophagus. Jpn J Lymphol. 1995;18:15-20 (in Japanese with English abstract).

19. Weinberg JA. The intrathoracic lymphatics. Lymphatics of the esophagus. In: Haagensen CD, Feind CR, Herter FP, Slantez CA, Weingberg JA, editors. Lymphatics in cancer. Philadelphia: WB Saunders; 1972. p. 245-9, 296-9.

20. Qubain SW, Natsugoe M, Matsumoto M, Nakashima S, Baba M, Takao S, Aikou T. Micrometastases in the cervical lymph nodes in esophageal squamous cell carcinoma. Dis Esophagus. 2001;14:143148. 